\title{
Analysis of High Radon-222 Concentration Events Using Multi-Horizontal-Resolution NICAM Simulations
}

\author{
Kentaro Ishijima ${ }^{1, *}$, Masayuki Takigawa ${ }^{1}$, Yousuke Yamashita ${ }^{1}$, Hisashi Yashiro ${ }^{2}$, Chihiro Kodama ${ }^{1}$, \\ Masaki Satoh ${ }^{3,1}$, Kazuhiro Tsuboi ${ }^{4}$, Hidekazu Matsueda ${ }^{4}$, Yosuke Niwa ${ }^{4, * *}$, and Shigekazu Hirao ${ }^{5}$ \\ ${ }^{1}$ Japan Agency for Marine-Earth Science and Technology, Yokohama, Japan \\ ${ }^{2}$ RIKEN Center for Computational Science, Kobe, Japan \\ ${ }^{3}$ Atmosphere and Ocean Research Institute, The University of Tokyo, Kashiwa, Japan \\ ${ }^{4}$ Meteorological Research Institute, Tsukuba, Japan \\ ${ }^{5}$ Institute of Environmental Radioactivity, Fukushima University, Fukushima, Japan
}

\begin{abstract}
Atmospheric radon-222 $\left({ }^{222} \mathrm{Rn}\right)$ variability is analyzed and compared with model simulations made by the Nonhydrostatic Icosahedral Atmospheric Model (NICAM), with three horizontal resolutions $(223,56$, and $14 \mathrm{~km})$, in order to understand high ${ }^{222} \mathrm{Rn}$ events predominantly caused by frontal activities. Seasonal variations of event frequency are well reproduced by the model, with correlation coefficients of $0.79(223 \mathrm{~km})$ to $0.99(14 \mathrm{~km})$. The three horizontal resolutions can reproduce general features of the observed peak shapes of events in winter, which dominantly reflect the passage of cold fronts that trap dense amounts of ${ }^{222} \mathrm{Rn}$. Peak height and width are well reproduced by the $56 \mathrm{~km}$ and 14 $\mathrm{km}$ resolution models, while the $223 \mathrm{~km}$ resolution model shows much lower and broader peaks due to insufficient resolution. We also find that simulations of ${ }^{222} \mathrm{Rn}$ and equivalent potential temperature gradient $\left(\left|\nabla \theta_{e}\right|\right)$ during the events show similar horizontal distributions around the ${ }^{222} \mathrm{Rn}$ observation station, suggesting $\left|\nabla \theta_{e}\right|$ is a useful tool to understand the variability of atmospheric components around fronts. Consequently, model with horizontal resolution of $56 \mathrm{~km}$ and $14 \mathrm{~km}$ can well simulate spatiotemporal variations of atmospheric components driven by frontal activities, while $223 \mathrm{~km}$ resolution is not enough to reproduce them.

(Citation: Ishijima, K., M. Takigawa, Y. Yamashita, H. Yashiro, C. Kodama, M. Satoh, K. Tsuboi, H. Matsueda, Y. Niwa, and S. Hirao, 2018: Analysis of high radon-222 concentration events using multi-horizontal-resolution NICAM simulations. SOLA, 14, 111-115, doi:10.2151/sola.2018-019.)
\end{abstract}

\section{Introduction}

Low-pressure systems and accompanied frontal activity play important roles in the transport of atmospheric components such as air pollutants, greenhouse gases, and aerosols (e.g., Sawa et al. 2007). In East Asia, cold fronts actively develop from winter to spring (e.g. Schemm et al. 2015) and contribute to two major types of transport processes (e.g. Itahashi et al. 2010): transport from the boundary layer (BL) to the free troposphere (FT) and transport within the BL. The former is transport of components from the BL of the warm sector ahead of the front to the FT along the warm conveyor belt (WCB), often evolving to subsequent long-range intercontinental transport by strong westerlies (e.g., Cooper et al. 2004). The latter is transport within the BL in the cold sector behind the front. During this process, pollutants emitted from surface sources tend to be drawn along the front by the

Corresponding author: Kentaro Ishijima, Japan Agency for Marine-Earth Science and Technology - Project Team for HPC Advanced Predictions utilizing Big Data, 3173-25 Showa-machi Kanazawa-ku, Yokohama, Kanagawa 236-0001 Japan. Email: ishijima@mri-jma.go.jp.

* Present affiliation: Meteorological Research Institute, Tsukuba, Japan

** Present affiliation: National Institute for Environmental Studies, Tsukuba, low-pressure system, trapped in the cold air, and predominantly transported by eastward migration of the front. Thus, elevated pollutant concentration is sometimes detected as a pollution event at surface monitoring stations (e.g. Sawa et al. 2007).

Fronts are generally mesoscale structures, but actually display a large variety of sizes: a few to tens of thousands of kilometers long and several to hundreds of kilometers wide (e.g., Sinclair et al. 2012; Schemm et al. 2015). Very narrow fronts are occasionally observed, represented in satellite images by narrow bands of rain or cloud $10-30 \mathrm{~km}$ wide and $\sim 1000 \mathrm{~km}$ long (rope cloud), which appear ahead of the front around the beginning or end of the frontal life (e.g., Kobayashi et al. 2007; Norris et al. 2017). Fine-scale analysis of phenomena around cold fronts typically requires a mesoscale weather prediction model (e.g., Kawashima 2016), while global climate models are often used for climatological analysis of fronts using simulations for several decades (e.g., Schemm et al. 2017). Atmospheric components are affected by fine-scale meteorological processes such as cumulus convection around fronts, sometimes leading to intercontinental transport through the WCB. Therefore, the ideal atmospheric chemistry transport model should be based on a global high-resolution cloud-resolving model. Yamaura et al (2013) used the Nonhydrostatic Icosahedral Atmospheric Model (NICAM) (Tomita and Satoh 2004; Satoh et al. 2008, 2014) with a global resolution of $14 \mathrm{~km}$, which can reproduce synoptic-scale phenomena and the life cycle of cumulus convection, to study the relationship between a tropical cyclone and the Baiu front in East Asia. Aizawa et al. (2014) realistically simulated Arctic fronts using NICAM with $14 \mathrm{~km}$ horizontal resolution. Sato et al. (2016) estimated, based on NICAM simulations with horizontal resolutions of 56, 14, and $3.5 \mathrm{~km}$, that black carbon is more efficiently transported upwards around low-pressure and frontal systems using a higher resolution NICAM, which can simulate fine atmospheric structure around the front. These studies are examples of an ideal atmospheric model successfully simulating synoptic to global scale transport processes.

Radon-222 $\left({ }^{222} \mathrm{Rn}\right)$ is a radioactive inert gas with a half-life of approximately 3.82 days. It is produced by decay of radium-226 $\left({ }^{226} \mathrm{Ra}\right)$, which occurs widely in soil particles and rocks on land; hence, almost all land masses are essentially a source of ${ }^{222} \mathrm{Rn}$. The emission rate from the ocean is smaller than that from soils by approximately two orders of magnitude (Wilkening and Clements 1975). Because of these characteristics, ${ }^{222} \mathrm{Rn}$ has been used to parameterize the transport of atmospheric components from land (e.g., Biraud et al. 2000) and evaluate model transport (e.g., Zhang et al. 2008; Belikov et al. 2013).

In this study, we compare the spatiotemporal variability of atmospheric ${ }^{222} \mathrm{Rn}$ during high concentration events, which are mainly caused by frontal activities, between observations and model simulations using NICAM with three horizontal resolutions. We discuss ${ }^{222} \mathrm{Rn}$ variability related to fronts based on climatological analyses. 


\section{Data and methods}

Brief descriptions of the observational data, model simulations, and analytical approaches are given here; more detailed explanations are described in Supplementary Material. We used continuous ${ }^{222} \mathrm{Rn}$ measurement data from Minamitorishima (hereafter MNM) (Meteorological Research Institute, Japan (Wada et al. 2012)) and Bermuda (BMW) (Environmental Measurement Laboratory (Hutter et al. 1995)), which are detailed in Fig. 1. For BMW, ${ }^{222} \mathrm{Rn}$ data influenced by local sources were excluded before analysis applying criteria by Hutter et al. (1995). We also used equivalent potential temperature $\left(\theta_{e}\right)$, which is widely used to locate fronts (e.g. Schemm et al. 2015). Large temporal change or horizontal gradient of $\theta_{e}$ tend to indicate the passage or location of the front, respectively. For analysis of time-series data of $\theta_{e}$ at MNM and BMW, direct measurement data at MNM station and NCEP North American Regional Reanalysis (NARR) data were used, respectively. For analysis of the two-dimensional meteorological fields, including $\theta_{e}$, the first model layer of ECMWF Interim reanalysis data (ERA-Interim, Dee et al. 2011), with a resolution of $0.75^{\circ} \times 0.75^{\circ}$, was used.

NICAM was used to simulate atmospheric ${ }^{222} \mathrm{Rn}$ concentration with three horizontal resolutions of 223, 56, and $14 \mathrm{~km}$ (hereafter referred to as $\Delta 223, \Delta 56$, and $\Delta 14$, respectively) and 38 vertical layers $(80-37000 \mathrm{~m})$. Although Niwa et al. (2011) simulated atmospheric ${ }^{222} \mathrm{Rn}$ using NICAM $(\Delta 223)$ nudged to reanalysis data, we did not nudge the model meteorology because it obscures the meteorological field produced by the model. We used a cumulus parameterization scheme by Chikira and Sugiyama (2010) for $\Delta 223$ and $\Delta 56$ simulations only. For cloud formation processes, a large-scale condensation scheme (Le Trent and Li 1991) was used for $\Delta 223$ and $\Delta 56$, while a cloud microphysics scheme (Tomita 2008) was used for $\Delta 14$.

The purpose of this study was to compare the resolution dependent differences of atmospheric structure produced by the model through an analysis of ${ }^{222} \mathrm{Rn}$ variability. We used monthly emission data by Hirao et al. (2010) for the ${ }^{222} \mathrm{Rn}$ simulations. Results of the contiguous free-run from 2009-2015 were used for the analyses. Detailed model simulation settings are described in Supplementary Material. To extract high ${ }^{222} \mathrm{Rn}$ events from the time-series data, an algorithm by Wada et al. (2012) was used. The method was slightly modified in this study, which uses the curve fitted to the observation data and the moving-average of the observation data, as shown in Fig. 2 (see Supplementary Material for more details).

We used the change rate of $\theta_{e}\left(\partial \theta_{e} / \partial t\right)$ to select events that are relatively more affected by fronts, since the $\partial \theta_{e} / \partial t$ is negative and decreases when a cold front passes. We averaged $\partial \theta_{e} / \partial t$ values within ten hours before and after the ${ }^{222} \mathrm{Rn}$ peak time for each event, and regarded the mean $\partial \theta_{e} / \partial t$ as an index of front strength for the event. Then, events whose mean $\partial \theta_{e} / \partial t$ was lower than the

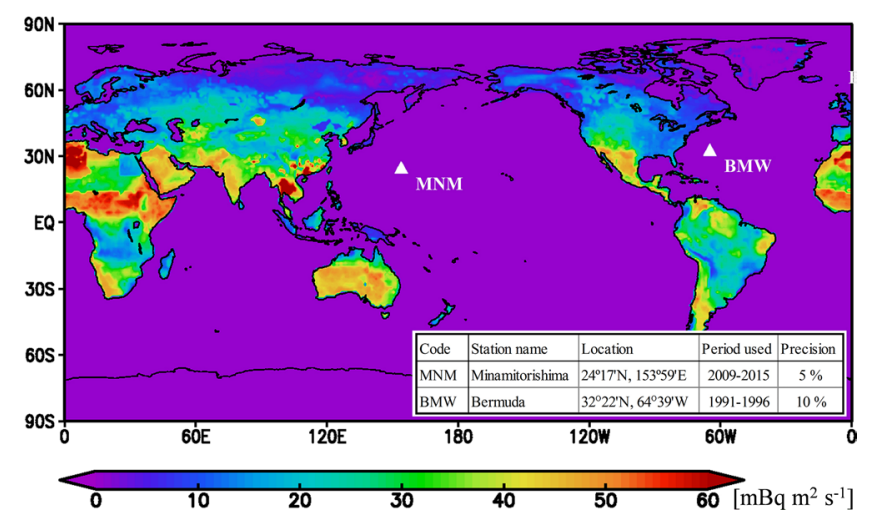

Fig. 1. ${ }^{222} \mathrm{Rn}$ emissions (mean for Dec, Jan, and Feb) used for atmospheric ${ }^{222} \mathrm{Rn}$ simulations and locations of ${ }^{222} \mathrm{Rn}$ observation stations used in this study. Detailed information of the observations is shown in the inset table.

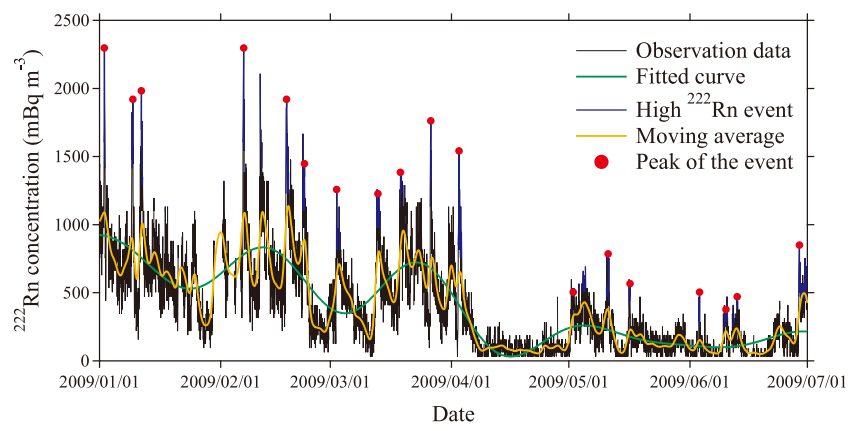

Fig. 2. ${ }^{222} \mathrm{Rn}$ concentrations observed at Minamitorishima station $(\mathrm{Obs})$, the curve fitted to $O b s$, detected high ${ }^{222} \mathrm{Rn}$ event, the moving average of $O b s$, and peak of the event.

$50^{\text {th }}$ percentile for all events were regarded as more front-affected, "selected events".

According to Aizawa et al. (2014), we also used the gradient of $\theta_{e}$ as an index of front strength on a two-dimensional field:

$$
\left|\nabla \theta_{e}\right|=\sqrt{\left(\frac{\partial \theta_{e}}{\partial x}\right)^{2}+\left(\frac{\partial \theta_{e}}{\partial y}\right)^{2}}
$$

We used the lowest layer data of model, corresponding to the altitude of the two observation stations, for analysis of $\left|\nabla \theta_{e}\right|$ and ${ }^{222} \mathrm{Rn}$.

\section{Results and discussion}

Figure 3 shows the frequency of high ${ }^{222} \mathrm{Rn}$ event peaks for each season, which were detected by applying the event detection algorithm to observed and simulated time-series data. Despite long-term free-runs, the model approximately shows consistent event frequencies with the observations within the error ranges, indicating realistic meteorology in the model. The maximum frequency tends to occur in the winter season (DJF) for both observation and model, as expected from Fig. 2 and from the fact that cold fronts most frequently occur in winter (Schemm et al. 2015). The overestimation of the frequency at MNM by higher resolution models might be related to larger $\theta_{e}$ changes by higher resolution models (Fig. 4), although it is difficult to untangle the cause within the scope of this study. The correlation coefficient of the seasonal variation of event frequency between model and observation typically exceeds 0.8 , indicating that the model can effectively reproduce the observed seasonal variation in high ${ }^{222} \mathrm{Rn}$ event frequency. It is clear that the higher resolution models exhibit better correlation coefficients, with $\Delta 14$ reaching 0.99 . These results suggest that, considering only the monthly changes of ${ }^{222} \mathrm{Rn}$ emissions are input in the models, high horizontal resolution NICAM is capable of realistically reproducing seasonal change tendency of ${ }^{222} \mathrm{Rn}$ transport around the stations predominantly caused by frontal activity. Based on these results, we hereafter focus on the winter season when high ${ }^{222} \mathrm{Rn}$ events are most frequent. Largest number of events $(\geq 45)$ is also an advantage for following climatological analyses based on the composite means.

Figure 4 shows the mean temporal variations of ${ }^{222} \mathrm{Rn}, \theta_{e}$, and $\partial \theta_{e} / \partial t$ for high ${ }^{222} \mathrm{Rn}$ events in winter. To determine the degree of ${ }^{222} \mathrm{Rn}$ increase during events relative to the base concentration level, the ratio of raw ${ }^{222} \mathrm{Rn}$ to the fitted-curve $\left({ }^{222} \mathrm{Rn}\right.$-ratio) is shown (corresponding to [black line]/[green line] in Fig. 2). In addition to the mean values for all events, the mean for "selected events" is also shown. The ${ }^{222} \mathrm{Rn}$-ratio shows an increase from the base line (fitted-curve) by a factor of 2.0 to 3.5 . ${ }^{222} \mathrm{Rn}$ decays less during transport from surface sources to the station in event periods than in non-event periods, due to the shorter transport 


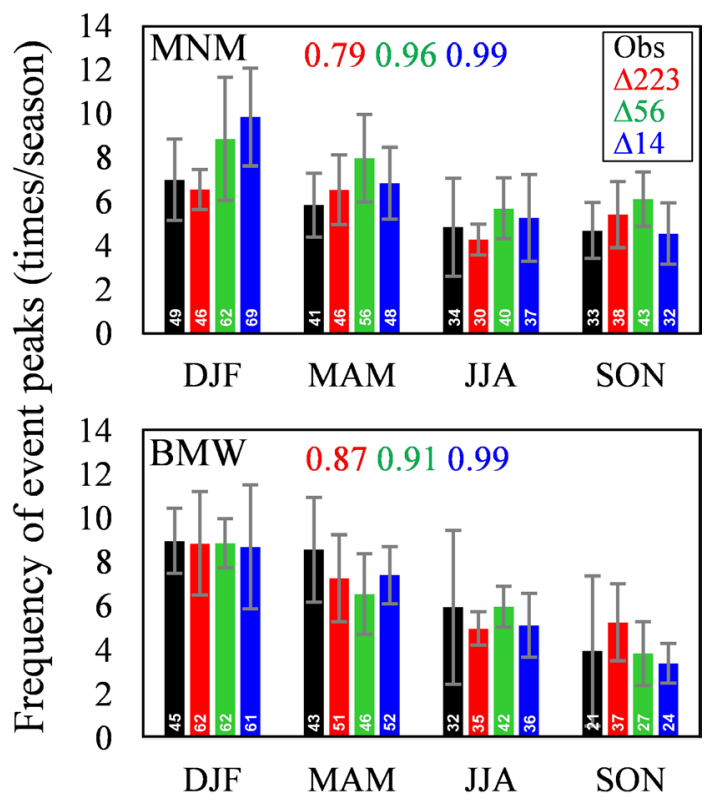

Fig. 3. Frequency of high ${ }^{222} \mathrm{Rn}$ event peaks for each season at each station. Error bars represent standard deviations $(1 \sigma)$ derived from the frequencies for individual years. Correlation coefficients between the seasonal changes of the frequency of observation $(O b s)$ and models $(\Delta 223, \Delta 56$, and $\Delta 14)$ are shown by numbers (indicated by red, green, and blue colors for $\Delta 223$, $\Delta 56$, and $\Delta 14$, respectively). Number of counts for high ${ }^{222} \mathrm{Rn}$ event peaks for each season is also shown in the bar, as information for statistical efficiency of climatological analyses in this study. time caused by stronger wind and/or shorter transport pathways. The major source regions are located in the west to northwest of the stations: the Sea of Japan side of Eurasia and the east side of North America for MNM and BMW, respectively (Fig. 1). Then, a cold front, which has trapped the continental-origin ${ }^{222} \mathrm{Rn}$ in the cold sector, migrates eastward or southeastward, passes over the station, and leads to a high ${ }^{222} \mathrm{Rn}$ event. At that time, ${ }^{222} \mathrm{Rn}$, which is densly accumulated along a band of large horizontal temperature gradient (just behind the front), simultaneously leads to maximum ${ }^{222} \mathrm{Rn}$ concentrations and a rapid decrease of $\theta_{e}$. Larger peaks and $\partial \theta_{e} / \partial t$ at BMW than at MNM could be related to the shorter distance from the continent, on which cold and high ${ }^{222} \mathrm{Rn}$ air exist, and the higher latitude. However, the correlation between the two components for all events at each station is not significant probably due to complex history of ${ }^{222} \mathrm{Rn}$ transport. Larger $\partial \theta_{e} / \partial t$ simulated by the model, especially at BMW, are due to the fact that cyclones, which develop around Newfoundland during event periods and bring cold continental air to BMW, tend to be stronger in NICAM than in ERA.

A comparison between observation and models shows that observed ${ }^{222} \mathrm{Rn}$-ratios have the sharpest peaks at both stations. The results are reasonable considering that a resolution of $<10 \mathrm{~km}$ is required to reproduce the detailed structure of low-pressure and frontal systems and associated transport (Sato et al. 2016). Peaks of $\Delta 223$ are the broadest and lowest at both stations, indicating that a horizontal resolution of $223 \mathrm{~km}$ is inadequate for reproducing observed ${ }^{222} \mathrm{Rn}$ peak sharpness as well as accurate frontal structure. The peak sharpness produced by $\Delta 56$ and $\Delta 14$ are almost comparable, but $\Delta 14$ reproduces the observed peak height better at MNM. These results suggest that the threshold of model horizontal resolution required to reasonably simulate the atmospheric structure around the cold front and produce realistic spa-
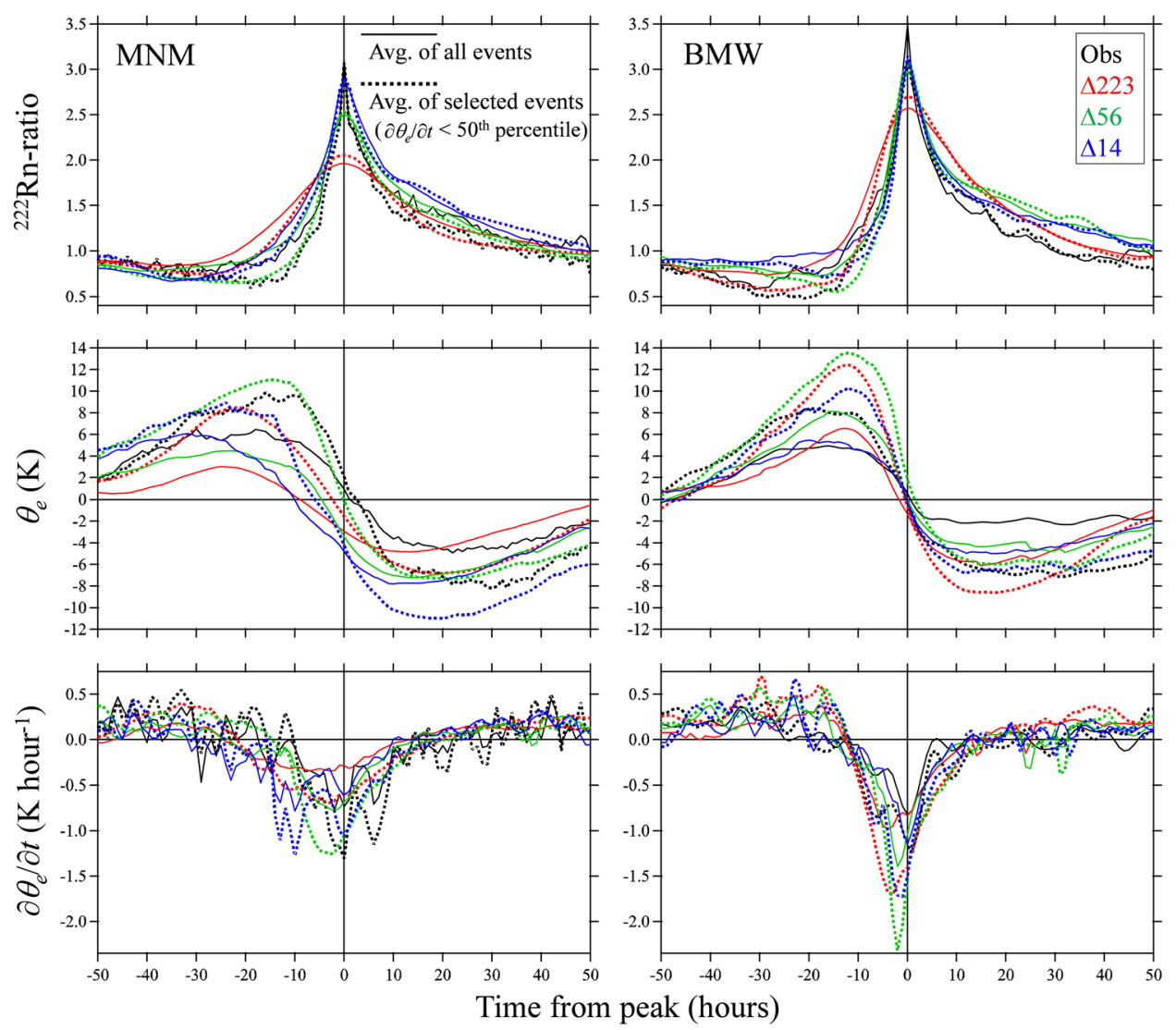

Fig. 4. Mean temporal variations of ${ }^{222} \mathrm{Rn}$-ratio, equivalent potential temperature $\left(\theta_{e}\right)$, and its temporal change rate $\left(\partial \theta_{e} / \partial t\right)$ for high ${ }^{222} \mathrm{Rn}$ events from the observations and model simulations. ${ }^{222} \mathrm{Rn}$-ratio is the ratio of the raw ${ }^{222} \mathrm{Rn}$ value to the value of the curve fitted to the raw data. $\theta_{e}$ is also fitted and the resudual is shown. Minus and plus values on the horizontal axis indicate the time before and after the ${ }^{222} \mathrm{Rn}$ peak of each event, respectively. "Obs" for $\theta_{e}$ at MNM and BMW represent the direct measurement at MNM station and the NCEP North American Regional Reanalysis data, respectively. 
tiotemporal ${ }^{222} \mathrm{Rn}$ variability is between 223 and $56 \mathrm{~km}$. For more details of the peak shape, the ${ }^{222} \mathrm{Rn}$-ratio increases rapidly before the peak, then slowly decreases, reaching a higher base level after the peak than that before the peak in both the observation and models. This temporal transition of ${ }^{222} \mathrm{Rn}$, which highlights the horizontal transition of ${ }^{222} \mathrm{Rn}$ across the frontal line and movement of the front itself, is well reproduced by all model resolutions. The fact is also supported by temporal variation of $\theta_{e}$, showing maximum decreasing rate at the time of or just before the ${ }^{222} \mathrm{Rn}$ peak. Given the maximum $\theta_{e}$ decrease point represents the frontal line, ${ }^{222} \mathrm{Rn}$ is supposed to be accumultaed along or behinde the cold front. For selected events (dashed lines in Fig. 4), the diminution of $\theta_{e}$ and the minimum of $\partial \theta_{e} / \partial t$ are predictably enhanced, by a factor of 1.5 to 2.0, compared to those of all events, but the factors show no clear relationship between observation and models and between stations. The ${ }^{222} \mathrm{Rn}$-ratio increase occurs later, or the slope before the peak is steeper than those of all events. These results are reasonable considering that stronger fronts (with larger $\partial \theta_{e} / \partial t$ around the ${ }^{222} \mathrm{Rn}$-ratio peak) result in larger horizontal ${ }^{222} \mathrm{Rn}$ gradients across the front.

To understand horizontal patterns of ${ }^{222} \mathrm{Rn}$ and related meteorological parameters during event periods, the mean horizontal distributions of those for selected events are shown in Fig. 5. Front strength indicated by $\left|\nabla \theta_{e}\right|$ (see "Data and method") are clearly enhanced over the ocean around stations in event periods, showing the maximum around the station. Intriguingly, the horizontal distribution of ${ }^{222} \mathrm{Rn}$-ratio is very similar to that of $\left|\nabla \theta_{e}\right|$ in model results, as expected from the relationship between ${ }^{222} \mathrm{Rn}$-ratio and $\partial \theta_{e} / \partial t$ in Fig. 4. This similarity tells that $\left|\nabla \theta_{e}\right|$ is a very important factor to understand the variability of atmospheric components around cold fronts. For MNM, ERA and the models show a common meteorological pattern of a low-pressure system located to the east of Hokkaido, Japan accompanied by a cold front, indicated by a band of enhanced $\left|\nabla \theta_{e}\right|$ over the station. The pattern is similar for BMW but the low-pressure system is located around Newfoundland. On the northwest side of the stations, northwest winds dominate during event periods, bringing ${ }^{222} \mathrm{Rn}$ from continental sources to the downstream stations. Southwest winds toward the low-pressure system in the south of and along the front also trap ${ }^{222} \mathrm{Rn}$ from the continent. Thus, ${ }^{222} \mathrm{Rn}$ accumulates strongly around the front. $\Delta 56$ shows that ${ }^{22} \mathrm{Rn}$ is dense behind the front around BMW (Fig. $5 \mathrm{~g}$ ), as also seen in temporal variations by $\Delta 56$ (Fig. 4), but such feature is not clear in other results. Over MNM, the strength of the front in $\Delta 223$ is weak $\left(\left|\nabla \theta_{e}\right|\right.$ $\sim 0.03 \mathrm{~K} \mathrm{~km}^{-1}$ ) compared to those in other results, although still shows the existence of the front as well as the accompanying ${ }^{222} \mathrm{Rn}$ increase. $\Delta 56$ and $\Delta 14$ show narrower bands around the stations than $\Delta 223$. The maximum $\left|\nabla \theta_{e}\right|$ and ${ }^{222} \mathrm{Rn}$-ratio are larger in higher resolution data, although the locations of the maximum points are not always precisely at the station. $\Delta 14$ shows several narrow branches of $\left|\nabla \theta_{e}\right|$ especially around MNM and very fine structures in the open ocean areas. Highly enhanced fronts, such as the arctic fronts reported by Aizawa et al. (2014) $\left(>\sim 0.2 \mathrm{~K} \mathrm{~km}^{-1}\right)$, sometimes occurs around both stations (not visible in Fig. 5) but only in $\Delta 14$. This seems to indicate the potential of such high resolution model to reproduce atmospheric component variability due to some extreme meteorological conditions, although such cases are not focused in this study.

\section{Summary}

We conducted model simulations of atmospheric ${ }^{222} \mathrm{Rn}$ concentration using NICAM with three horizontal resolutions of 223,56 , and $14 \mathrm{~km}$ and compared them with observational data of high ${ }^{222} \mathrm{Rn}$ events predominantly caused by frontal activities. Climatological analysis was applied and the seasonal variations of event frequency were well reproduced by the models. The correlation coefficients between models and observations were predominantly over 0.8 , and reached 0.99 for $\Delta 14$, indicating the better performance of higher resolution NICAM for simulating synoptic ${ }^{222} \mathrm{Rn}$ transport around the stations. Temporal variations of ${ }^{222} \mathrm{Rn}$-ratio during the event in winter were well reproduced by all resolutions of the models in terms of the rate of change or the level before and after the peak. The observed event peak sharpness was well reproduced by $\Delta 56$ and $\Delta 14$, while $\Delta 223$ showed a much lower and broader peak due to the coarse horizontal resolution. This indicates that $223 \mathrm{~km}$ horizontal resolution is completely inadequate to reasonably simulate atmospheric structure as well as contrast of ${ }^{222} \mathrm{Rn}$ distributions around fronts. We found that model simulations of ${ }^{222} \mathrm{Rn}$ and the equivalent potential temperature gradient (indicator of front strength) for the events showed very similar horizontal distributions. The results show that the use of $\left|\nabla \theta_{e}\right|$ is helpful to understand the variability of atmospheric components around fronts.

This study indicates that higher resolution models capable of simulating finer scale atmospheric structure are more profitable for atmospheric component studies. Such models also could more
ERA

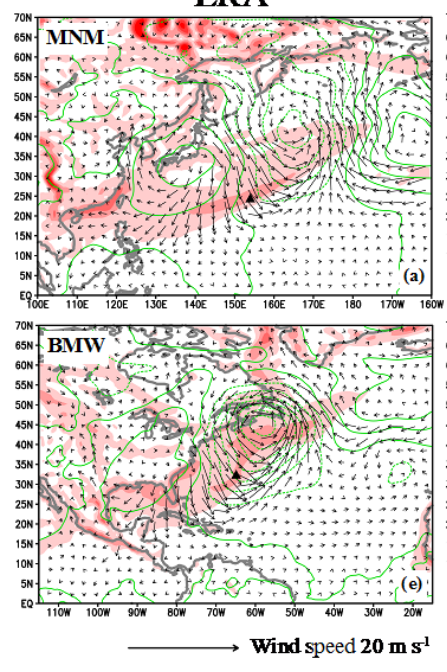

$\Delta 223$
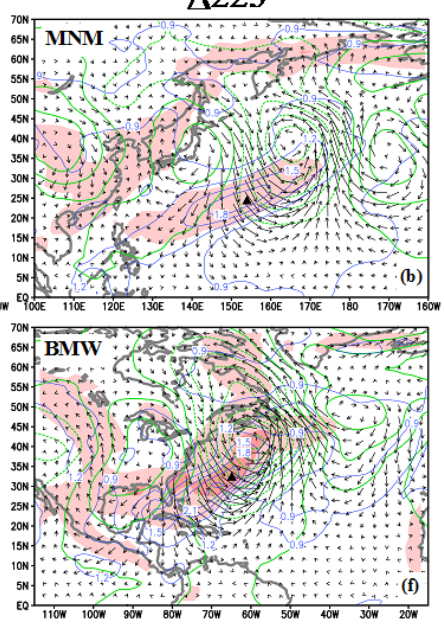

$\triangle 56$
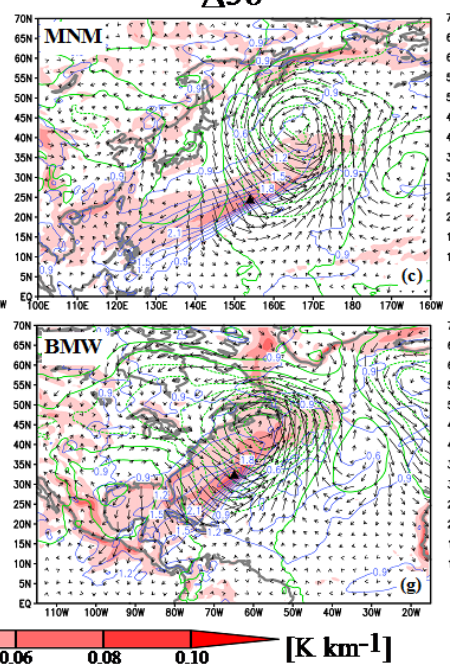

$\Delta 14$
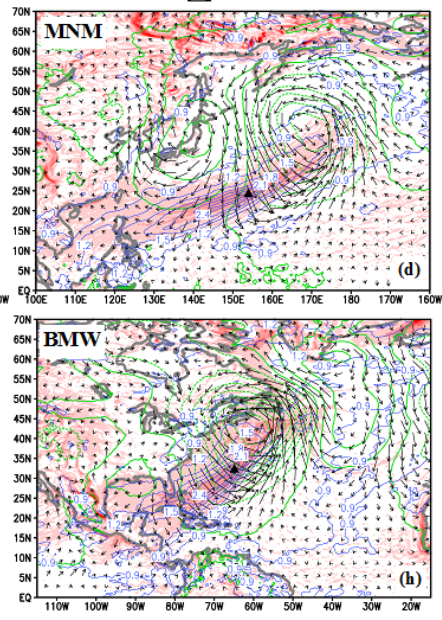

Fig. 5. Gradient of composite mean equivalent potential temperature $\left(\theta_{e}\right)$ (red color) and the composite means of wind (vector), of mean sea level pressure (green contour), and of the ${ }^{222} \mathrm{Rn}$-ratio (blue contour, for only model) for selected events $\left(\partial \theta_{e} / \partial t<50\right.$ th percentile) in winter around $\mathrm{MNM}$ (a-d) and BMW $(\mathrm{e}-\mathrm{h})$ from ERA-Interim (ERA) and model simulations $(\Delta 223, \Delta 56$, and $\Delta 14)$. Deviation from the seasonal mean is shown for winds and mean sea level pressure, while the ratio to the seasonal mean is shown for the ${ }^{222} \mathrm{Rn}$-ratio. Station locations are indicated by black closed triangles. 
precisely reflect observational data, sometimes affected by fine scale meteorological phenomena, in the fluxes estimated by data assimilation or inverse modeling than low-resolution models. In this regard, more detailed analysis to reveal the four-dimensional structure of atmospheric components driven by frontal activities or tropical cyclones and subsequent global-scale transport using a high-resolution global model would contribute substantially to atmospheric environmental studies.

\section{Acknowledgements}

The authors are grateful to anonymous reviewers for their constructive comments. This work was supported by the FLAGSHIP2020, MEXT, within priority study 4 (advancement of meteorological and global environmental predictions utilizing observational "Big Data"). The NICAM calculation was performed using the K computer at the RIKEN R-CSS as part of the HPCI System Research project (Project ID: hp160231, hp170232).

Edited by: D. Goto

\section{References}

Aizawa, T., H. L. Tanaka, and M. Satoh, 2014: Rapid development of Arctic cyclone simulated by the cloud resolving global model NICAM. Meteor. Atmos. Phys., 126, 105-117.

Belikov, D. A., and co-authors, 2013: Off-line algorithm for calculation of vertical tracer transport in the troposphere due to deep convection. Atmos. Chem. Phys., 13, 1093-1114.

Biraud, S., P. Ciais, M. Ramonet, P. Simmonds, V. Kazan, P. Monfray, S. O'Doherty, T. G. Spain, and S. G. Jennings, 2000: European greenhouse gas emissions estimated from continuous atmospheric measurements and radon 222 at Mace Head, Ireland. J. Geophys. Res., 105, 1351-1366.

Chikira, M., and M. Sugiyama, 2010: A cumulus parameterization with state-dependent entrainment rate. Part I: Description and sensitivity to temperature and humidity profiles. $J$. Atmos. Sci., 67, 2171-2193.

Cooper, O. R., C. Forster, D. Parrish, M. Trainer, E. Dunlea, T. Ryerson, G. Hubler, F. Fehsenfeld, D. Nicks, J. Holloway, J. de Gouw, C. Warneke, J. M. Roberts, F. Flocke, and J. Moody, 2004: A case study of transpacific warm conveyor belt transport: Influence of merging airstreams on trace gas import to North America. J. Geophys. Res., 109, D23S08, doi:10.1029/2003JD003624.

Dee, D. P., and co-authors, 2011: The ERA-Interim reanalysis: Configuration and performance of the data assimilation system. Quart. J. Roy. Meteor. Soc., 137, 553-597.

Hirao, S., H. Yamazawa, and J. Moriizumi, 2010: Estimation of the global ${ }^{222} \mathrm{Rn}$ flux density from the Earth's surface. $J$. Health Phys. Japan, 45, 161-171.

Hutter, A. R., R. J. Larsen, H. Maring, and J. T. Merrill, 1995: ${ }^{222} \mathrm{Rn}$ at Bermuda and Mauna Loa: Local and distant sources. J. Radioanal. Nucl. Chem., 193, 309-318.

Itahashi, S., K. Yumimoto, I. Uno, K. Eguchi, T. Takemura, Y. Hara, A. Shimizu, N. Sugimoto, and Z. Liu, 2010: Structure of dust and air pollutant outflow over East Asia in the spring. Geophys. Res. Lett., 37, L20806, doi:10.1029/2010 GL044776.

Kawashima, M., 2016: The role of vertically propagating gravity waves forced by melting-induced cooling in the formation and evolution of wide cold-frontal rainbands. Bull. Amer. Meteor. Soc., 73, 2803-2836.

Kobayashi, F., Y. Sugawara, M. Imai, M. Matsui, A. Yoshida, and
Y. Tamura, 2007: Tornado generation in a narrow cold frontal rainband -Fujisawa tornado on April 20, 2006-. SOLA, 3, 21-24.

Le Trent, H., and Z. X. Li, 1991: Sensitivity of an atmospheric general circulation model to prescribed SST changes: Feedback effects associated with the simulation of cloud optical properties. Climate Dyn., 5, 175-187.

Niwa, Y., H. Tomita, M. Satoh, and R. Imasu, 2011: A threedimensional icosahedral grid advection scheme preserving monotonicity and consistency with continuity for atmospheric tracer transport. J. Meteor. Soc. Japan, 89, 255-268.

Norris, J., G. Vaughan, and D. M. Schultz, 2017: Variability of precipitation along cold fronts in idealized baroclinic waves. Mon. Wea. Rev., 145, 2971-2992.

Sato, Y., H. Miura, H. Yashiro, D. Goto, T. Takemura, H. Tomita, and T. Nakajima, 2016: Unrealistically pristine air in the Arctic produced by current global scale models. Sci. Rep., 6, 26561, doi:10.1038/srep26561.

Satoh, M., T. Matsuno, H. Tomita, H. Miura, T. Nasuno, and S. Iga, 2008: Nonhydrostatic icosahedral atmospheric model (NICAM) for global cloud resolving simulations. J. Comp. Phys., 227, 3486-3514.

Satoh, M., and co-authors, 2014: The non-hydrostatic icosahedral atmospheric model: Description and development. Prog. Earth Planet. Sci., 1, 2293, doi:10.1186/s40645-014-0018-1.

Sawa, Y., and co-authors, 2007: Widespread pollution events of carbon monoxide observed over the western North Pacific during the East Asian Regional Experiment (EAREX) 2005 campaign. J. Geophys. Res., 112, D22S26, doi:10.1029/ 2006JD008055.

Schemm, S., I. Rudeva, and I. Simmonds, 2015: Extratropical fronts in the lower troposphere-Global perspectives obtained from two automated methods. Quart. J. Roy. Meteor. Soc., 141, 1686-1698.

Schemm, S., M. Sprenger, O. Martius, H. Wernli, and M. Zimmer, 2017: Increase in the number of extremely strong fronts over Europe? A study based on ERA-Interim reanalysis (1979-2014). Geophys. Res. Lett., 44, 553-561.

Sinclair, V. A., S. Niemelä, and M. Leskinen, 2012: Structure of a narrow cold front in the boundary layer: Observations versus model simulation. Mon. Wea. Rev., 140, 2497-2519.

Tomita, H., and M. Satoh, 2004: A new dynamical framework of nonhydrostatic global model using the icosahedral grid. Fluid Dyn. Res., 34, 357-400.

Tomita, H., 2008: New microphysical schemes with five and six categories by diagnostic generation of cloud ice. J. Meteor. Soc. Japan, 86A, 121-142.

Yamaura, T., Y. Kajikawa, H. Tomita, and M. Satoh, 2013: Possible impact of a tropical cyclone on the northward migration of the baiu frontal zone. SOLA, 9, 89-93.

Wada, A., H. Matsueda, S. Murayama, S. Taguchi, A. Kamada, M. Nosaka, K. Tsuboi, and Y. Sawa, 2012: Evaluation of anthropogenic emissions of carbon monoxide in East Asia derived from the observations of atmospheric radon-222 over the western North Pacific. Atmos. Chem. Phys., 12, 12119-12132.

Wilkening, M. H., and W. E. Clements, 1975: Radon-222 from the ocean surface. J. Geophys. Res., 80, 3828-3830.

Zhang, K., H. Wan, M. Zhang, and B. Wang, 2008: Evaluation of the atmospheric transport in a GCM using radon measurements: sensitivity to cumulus convection parameterization. Atmos. Chem. Phys., 8, 2811-2832.

Manuscript received 25 April 2018, accepted 25 June 2018 SOLA: https://www.jstage.jst.go.jp/browse/sola/ 